\title{
Cost- and Energy-Efficient Aerial Communication Networks with Interleaved Hovering and Flying
}

\author{
Nithin Babu, Student Member, IEEE, Marco Virgili, Student Member, IEEE, \\ Constantinos. B Papadias, Fellow, IEEE, Petar Popovski, Fellow, IEEE, and \\ Andrew Forsyth, Senior member, IEEE
}

\begin{abstract}
This work proposes a methodology for the energyand cost-efficient 3-D deployment of an unmanned aerial vehicle (UAV)-based aerial access point (AAP), that exchanges a given amount of independent data with a set of ground user equipment (UE). Considering a fly-hover-communicate transmission scheme, the most energy-efficient 3-D hovering points (HPs) of the AAP are determined by decoupling the problem in the horizontal and vertical dimensions. First, we derive analytically the optimal hovering altitude that jointly maximizes the downlink and uplink global energy efficiency (GEE) of the system. Next, we propose the multilevel circle packing (MCP) algorithm to determine the minimal number of HPs and their associated horizontal coordinates, such that the AAP covers all the UEs in the given geographical area. A cost analysis is carried out to observe the variation of both fixed and variable costs; these are then minimized by suitably selecting the AAP's battery parameters, like the depth of discharge (DOD), defined as the portion of battery capacity that is consumed during a discharge cycle, and the velocity of the UAV. Simulation results show that: the UAV energy consumption has a significant impact on the 3-D HPs of the AAP; the time spent during the substitution swap of an out of power AAP has a major influence on the operational cost; the cost of the system can be optimized by suitably selecting the onboard battery and the UAV flight parameters.
\end{abstract}

Index Terms - cost-optimization, energy-efficiency, 3-D placement optimization, UAV communication.

\section{INTRODUCTION}

$\mathbf{T}$ HE use of unmanned aerial vehicles (UAVs) deployed for providing temporary telecommunication services in a disaster-affected area or a special situation event is considered as an important application of the 5G and beyond technology [1], [2]. The mobile feature of UAV-based communication systems provides opportunities for better communication channels to the UEs as compared to conventional systems. The improved channel gain is due to the higher line-of-sight ( $\mathrm{LoS})$

Manuscript submitted on November 21, 2020 . This work is supported by the project PAINLESS which has received funding from the European Union's Horizon 2020 research and innovation programme under grant agreement No 812991. Copyright (c) 2015 IEEE. Personal use of this material is permitted. However, permission to use this material for any other purposes must be obtained from the IEEE by sending a request to pubs-permissions@ieee.org.

N. Babu and C. B. Papadias are with Research, Technology and Innovation Network (RTIN), Alba, The American College of Greece, Greece (e-mail: nbabu@acg.edu, cpapdias@acg.edu).

N. Babu, C. B. Papadias, and P. Popovski are with Department of Electronic Systems, Aalborg University, Denmark (e-mail: niba@es.aau.dk, copa@es.aau.dk, petarp@es.aau.dk).

M. Virgili is with Lyra Electronics and The University of Manchester (email: marco@lyraelectronics.com, marco.virgili@postgrad.manchester.ac.uk).

M. Virgili and A. Forsyth are with The University of Manchester (e-mail: marco.virgili@postgrad.manchester.ac.uk, andrew.forsyth@manchester.ac.uk). probability [4], shorter distance between the aerial access point (AAP) and the pieces of user equipment (UEs), and ability to choose a preferred link. On the other hand, the service time of a UAV-based AAP is determined by the available onboard energy. Due to this energy constraint, the deployment of the AAP should be done in an energy-efficient way to maximize the number of bits transmitted per Joule of energy consumed, as captured by the global energy efficiency (GEE) of the network [3]. As defined in [3], the GEE of an aerial communication network is the ratio of the sum rate in bits per second to the total power consumed. The total power consumed is the sum of the communication-related power and the power required by the UAV for flying and hovering.

The placement optimization of a UAV-based base station with the objective of optimizing communication-related parameters, such as sum rate and coverage, is well investigated in the literature. For instance, the authors of [4] present an analytical approach to optimize the altitude of a UAVbased base station for a given maximum allowed path loss. A joint altitude and beam-width optimization for throughput maximization is considered in [5]. A method to find the optimal 3-D location of a UAV base station is developed in [6], which maximizes the coverage region and optimizes the transmission power. In [7], the authors have developed a learning-based Intent-aware Upper Confidence Bound (IUCB) algorithm that could be used for offloading tasks in an airground integrated vehicular edge computing system. Works studying the deployment of a multi-UAV system for optimal wireless coverage and throughput maximization include [9], [10]. The work in [8] maximizes the revenue of the mobile crowd-sensing (MCS) carrier and the UAV via a joint optimization of route planning and task assignment subject to practical constraints of battery capacity and sensing latency. A 3-D deployment plan for a flying base station that serves the users according to their service requirements is presented in [11]. The placement optimization of dynamic standalone drones equipped with a steerable antenna is proposed in [12].

In [13], the authors maximize the minimum average rate and individual uplink energy-efficiency of multiple ground nodes supported by a UAV. The works [17], [18] maximize the downlink GEE of a UAV-based communication system flying at a constant altitude using the sequential convex programming-based trajectory optimization techniques. The trajectory optimizations considered in [14], [15], and [16] maximize the downlink throughput. In our previous work [3], we have determined the energy-efficient hovering altitude for a 
standalone AAP deployed for orthogonal downlink broadcast transmission. In another work [19], we have optimized the 3-D locations of a multi-UAV system which maximize the uplink GEE of the system in the presence of inter-cell interference. A detailed survey on the works that consider energy-efficient UAV deployment is available in [20].

Besides the placement optimization of the AAP, cost minimization is of great importance for the effective deployment of the aerial communication network. In order to minimize the installation cost of UAVs and charging stations, an efficient mixed-infrastructure placing was proposed in [21]. In [22], an economic analysis aimed at optimizing both coverage and capital cost is carried out, while [23] provides a novel technical solution for a sensor network and provides information on the price of all its components. However, the capital cost is just one part of the total expenses, which depend on how the system operates: parameters such as the number of UAVs, the UAV swap time, and the depth of discharge (DOD) need to be optimized.

The works [4]- [16] position UAVs for optimizing communication-related parameters such as sumrate, transmit power, or coverage region without considering the energyconsumption factors of the UAVs. [13], [17], and [18] maximize the GEE by considering a simple line-of-sight (LoS) single UAV system. Furthermore, from [20], none of the existing works, to the best of our knowledge, have considered a UAV placement problem that jointly maximizes the uplink and downlink GEE by considering both the communication and UAV-related energy consumption factors. Moreover, the UAV energy consumption models used are not directly applicable to a multi-rotor UAV-based system. In addition to this, operational cost minimization by optimizing the system parameters of a UAV network has, to the best of our knowledge, not yet been considered in the literature.

\section{A. Main Contributions and Paper Organization}

The work proposes an energy- and cost-efficient rotarywing UAV-assisted aerial communication network deployed to provide temporary service to a set of ground UEs. The main contributions are:

- We propose and use hovering and flying power consumption models that apply to any multi-rotor UAV. The power consumption models used in [17] cannot be applied directly to a multi-rotor UAV since they are based on the axial momentum theory applied to a single-rotor UAV.

- The altitude and beamwidth optimization considering both the communication-related and UAV energy consumption factors that maximize both the uplink and the downlink GEE has, to the best of our knowledge, not been investigated in the literature.

- A general framework for the optimal horizontal positioning of UAV in a target area is proposed through the polynomial-time complex multi-level circle packing (MCP) algorithm. The algorithm applies to the maximization of different objectives in which the coverage radius depends on the objective function to be maximized.

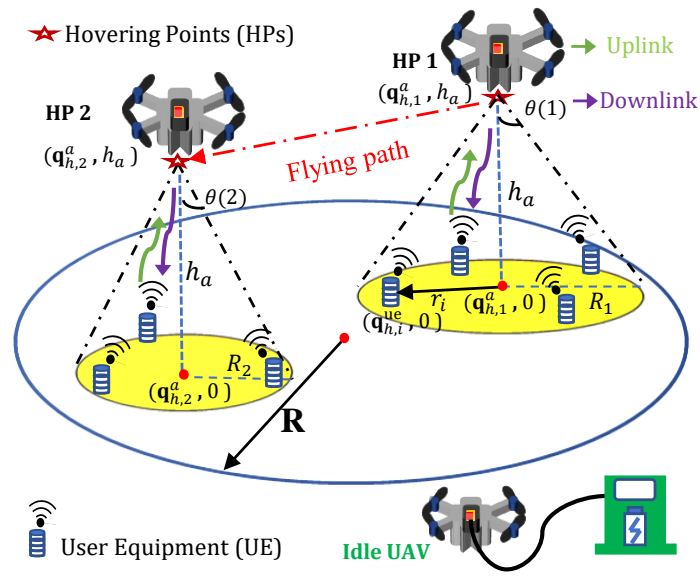

Fig. 1: System setup.

We consider the GEE as the performance matrix and determine the UAV coverage radius which maximizes both the uplink and downlink GEE of the system.

- A detailed economic analysis of the aerial network is provided; furthermore, the operational cost minimization by optimizing the number of UAVs, the UAV swap time, the UAV flying velocity, and the depth of discharge of the UAV battery has, to the best of our knowledge, not yet been considered in the literature.

Section III describes the system setup and assumptions taken for this study, while in subsection [II-C, we derive the expression to estimate the power consumed by a multi-rotor UAV during its hovering, horizontal and vertical movements. The optimal altitude and beamwidth that maximize both the uplink and the downlink GEE are determined in Section III-A and Section III-A1 respectively. Using the optimal altitude and beamwidth determined from Section III-A in Section III-B we propose a general framework for determining the minimum number and location of hovering points (HPs) to cover all the UEs in a given geographical region, using a polynomial-time complex MCP algorithm. The different factors that affect the deployment and operational cost of the considered network are discussed in Section IV The annualized cost of the system is minimized by suitably selecting the onboard battery parameter and the optimal horizontal flying velocity of the UAV. A detailed discussion of the main findings and the future scopes of work are provided in Sections $\mathrm{V}$ and $\mathrm{VI}$. In this paper, boldface lowercase letters are used to denote vectors.

\section{System Modelling AND Definitions}

We consider a delay-tolerant network consisting of a set of uniformly distributed UEs with a density of $\lambda_{\mathrm{u}} \mathrm{UEs} / \mathrm{m}^{2}$ over a circular geographical area of radius $R \mathrm{~m}$. We assume that each UE needs to send/receive data bits to/from the AAP every $T_{\mathrm{n}}$ seconds (s). Our objective is to deploy a single moving AAP in an energy- and cost-efficient way so that all

\footnotetext{
${ }^{1}$ This work considers the GEE HPs determination problem and the DOD optimization problem not jointly, but sequentially; the later one takes the set of energy-efficient HPs as the input to find the cost-efficient DOD.
} 
the UEs are served by the AAP once every $T_{\mathrm{n}} \mathrm{s}$. In order to do so, the AAP will follow a fly-hover-communicate protocol, meaning it will fly from one HP to another, hovers for the time needed to exchange the data packets, then moves on to the next destination as shown in Fig. 1. The AAP is deployed to serve the UEs for $t_{\text {miss }}$ hrs/day and, if the active time $\left(t_{\text {active }}\right)$ of the serving-UAV is less than $t_{\text {miss }}$, then the idle UAV will replace the out-of-power UAV while the latter descends to recharge.

We assume the AAP is equipped with a directional antenna with the antenna gain in the direction $(\epsilon, \kappa$,$) given by,$

$$
G_{\mathrm{a}}=\left\{\begin{array}{cc}
\frac{G_{\mathrm{o}}}{\theta^{2}} & -\theta \leq \epsilon \leq \theta,-\theta \leq \kappa \leq \theta, \\
g \approx 0 & \text { otherwise }
\end{array}\right.
$$

where $G_{\mathrm{o}} \approx 2.2846$ [5], and since the side lobe gain of the antenna is assumed very small compared to the main lobe gain, we can also assume $g \approx 0$. The half-power beamwidth of the antenna in the elevation and the azimuth plane is $2 \theta$. The UEs are equipped with an omnidirectional antenna of unitary gain. Hence the coverage area of a single AAP hovering at an altitude $h_{a}$ will be a circular region of radius $R_{\mathrm{a}}=h_{\mathrm{a}} \tan \theta$ as shown in Fig. 1. In addition, we assume an orthogonal multiple access transmission scenario between the AAP and its served UEs, where each UE in the geographical area is allocated a fixed bandwidth (e.g., in a narrowband frequency division multiple access system [26]).

The line-of-sight (LoS) and non-LoS (NLoS) air to ground channel links are considered to model the probabilistic mean path loss between the AAP and the UEs [4], given by [19]:

$$
\begin{aligned}
\bar{L}_{i} & =P_{1}\left(\phi_{i}\right) \times \frac{d_{i}^{2} \eta_{1}^{2}}{g_{\mathrm{o}}}+\left(1-P_{1}\left(\phi_{i}\right)\right) \times \frac{d_{i}^{2} \eta_{\mathrm{nl}}^{2}}{g_{\mathrm{o}}}, \\
& =\frac{d_{i}^{2}}{g_{\mathrm{o}}} \underbrace{\left[P_{1}\left(\phi_{i}\right)\left(\eta_{1}^{2}-\eta_{\mathrm{nl}}^{2}\right)+\eta_{\mathrm{nl}}^{2}\right]}_{L_{\mathrm{m}}\left(\phi_{i}\right)},
\end{aligned}
$$

where $\left.P_{1}\left(\phi_{i}\right)=1 /\left\{1+a \exp \left[-b\left(\phi_{i}-a\right)\right)\right]\right\}$ is a modified Sigmoid function that closely represents the LoS probability between the AAP and the $i^{t h}$ UE located at a distance of $r_{i}$ from the center of the AAP coverage area, corresponding to an elevation angle of $\phi_{i}=(180 / \pi) \tan ^{-1}\left(h_{\mathrm{a}} / r_{i}\right)$ with the AAP hovering at an altitude $h_{\mathrm{a}}$; the parameters $a$ and $b$ are directly linked to the environment variables such as the mean number of buildings, their height distribution, and the ratio of built-up land area to the total land area using the two variable surface fitting [4]; $g_{\mathrm{o}}$ is the channel gain at a reference distance of $1 \mathrm{~m} ; d_{i}=\sqrt{r_{i}^{2}+h_{\mathrm{a}}^{2}}$. Since the work considers the planning phase of the AAP deployment, we consider long term channel random variation rather than the small scale fading component [4], [6], [19]; $\eta_{\mathrm{l}}$ and $\eta_{\mathrm{nl}}$ are the mean values of the excess loss due to the man-made structures associated with the LoS and NLoS links, respectively.

\section{A. Downlink data rate}

Consider an AAP deployed to send independent information to a set of UEs through orthogonal channels be hovering at the HP1 position as shown in Fig. 1. The equivalent average SNR defined as the ratio of average received signal power to the noise power, received by a UE located at a distance of $r_{i}$ from the center of the coverage region is $\gamma_{i}^{\mathrm{d}}=\frac{G_{\mathrm{a}} P_{i}^{\mathrm{d}}}{\Gamma_{i} \sigma^{2} \bar{L}_{i}}$ $\Gamma_{i} \geq 1$ represents the gap between the channel capacity and the achievable rate due to the practical coding scheme and modulation scheme used; $\sigma^{2}$ is the variance of the zero-mean additive white Gaussian noise; $P_{i}^{\mathrm{d}}$ is the power allocated for the $i^{t h}$ UE. Hence the corresponding achievable data rate of the UE in bits per second is given by:

$$
S_{i}^{\mathrm{d}}=B \log _{2}\left(1+\gamma_{i}^{\mathrm{d}}\right) \text {, }
$$

where $B$ is the bandwidth allocated to each UE. The average sum rate, $\bar{S}_{\mathrm{d}}$, defined as the expectation of the sum of the achievable rate by all the UEs in the AAP coverage area is obtained by taking an expectation over the uniformly distributed UEs with a density $\lambda_{\mathrm{u}}$ :

$$
\bar{S}_{\mathrm{d}}=B \lambda_{\mathrm{u}} \int_{0}^{R_{\mathrm{a}}} 2 \pi S_{i}^{\mathrm{d}} r_{i} d r_{i},
$$

where $R_{\mathrm{a}}=h_{\mathrm{a}} \tan \theta$. The integral in (5) is difficult to evaluate because of the excess path loss factor, $L_{\mathrm{m}}\left(\phi_{i}\right)$. Since the LoS probability is a decreasing function of $r_{i}$, we approximate $P_{1}\left(\phi_{i}\right) \approx P_{1}\left(\phi_{\text {edge }}\right) \forall i$, where $P_{1}\left(\phi_{\text {edge }}\right)$ is the LoS probability of the edge UE [19] with $\phi_{\text {edge }}=90^{\circ}-\theta$ (in deg). Thus the average rate value evaluated using (5) with $L_{\mathrm{m}}\left(\phi_{i}\right)=L_{\mathrm{m}}\left(\phi_{\text {edge }}\right) \forall i$ is the lower bound of the actual rate value expressed as,

$$
\begin{aligned}
\bar{S}_{\mathrm{d}} & =2 \pi \lambda_{\mathrm{u}} B \int_{0}^{R_{\mathrm{a}}} \log _{2}\left(1+\frac{G^{\prime}}{\left(r_{i}^{2}+h_{\mathrm{a}}^{2}\right)}\right) r_{i} d r_{i}, \\
& =B^{\prime}\left(G^{\prime}+h_{\mathrm{a}}^{2} \sec ^{2} \theta\right) \log \left(G^{\prime}+h_{\mathrm{a}}^{2} \sec ^{2} \theta\right) \\
& -B^{\prime}\left(G^{\prime}+h_{\mathrm{a}}^{2}\right) \log \left(G^{\prime}+h_{\mathrm{a}}^{2}\right) \\
& -B^{\prime}\left[\left(h_{\mathrm{a}}^{2} \sec ^{2} \theta\right) \log \left(h_{\mathrm{a}}^{2} \sec ^{2} \theta\right)-h_{\mathrm{a}}^{2} \log \left(h_{\mathrm{a}}^{2}\right)\right],
\end{aligned}
$$

where $G^{\prime}=\frac{G_{\mathrm{a}} P_{i}^{\mathrm{d}} g_{\mathrm{o}}}{\Gamma_{i} \sigma^{2} L_{\mathrm{m}}(\theta)}, B^{\prime}=B \pi \lambda_{\mathrm{u}} \log _{2} e$.

\section{B. Uplink data rate}

A different deployment scenario in which the AAP hovering at HP1 of Fig. 1 is deployed to collect independent data from a set of ground UEs is considered in this section. We consider the uplink power control implemented according to the 3GPP technical report [24], through which each UE chooses its uplink transmit power so that the received SNRs at the AAP from all the UEs in the coverage region are equal. Hence, the average transmit power chosen by a UE located at a distance of $d_{i}$ from the AAP location in the uplink power control scheme is represented in its basic form as $P_{i}^{\mathrm{u}}=P_{\mathrm{a}} \bar{L}_{i}$; where $P_{\mathrm{a}}$ is the target power to be received at the AAP. Because of the uplink power control, the received SNR from all the UEs will be the same: $\gamma_{i}^{\mathrm{u}}=\frac{G_{\mathrm{a}} P_{\mathrm{a}}}{\Gamma_{i} \sigma^{2}}$ and the uplink data rate will be different from the downlink data rate:

$$
S_{i}^{\mathrm{u}}=B \log _{2}\left(1+\gamma_{i}^{\mathrm{u}}\right) .
$$

In addition to this, all the UEs lying in the coverage area of the AAP will have the same data rate given by (8); hence the sum of the data rate is given by,

$$
\bar{S}_{\mathrm{u}}=B \pi \lambda_{\mathrm{u}} h_{\mathrm{a}}^{2} \tan ^{2} \theta S_{i}^{\mathrm{u}} .
$$


TABLE I: UAV Parameters [30].

\begin{tabular}{lll}
\hline Label & Definition & Value \\
\hline \hline$W$ & Weight of the UAV in Newton & $35.28 \mathrm{~N}$ \\
$N_{\mathrm{R}}$ & Number of rotors & 4 \\
$F_{\mathrm{n}}$ & Upward thrust by the $n^{\text {th }}$ rotor & - \\
$v$ & UAV's horizontal flying velocity & - \\
$v_{\text {tip }}$ & Tip speed of the rotor & $102 \mathrm{~m} / \mathrm{s}$ \\
$A_{\mathrm{f}}$ & Fuselage area & $0.2113 \mathrm{~m}^{2}$ \\
$\rho\left(h_{a}\right)$ & Air density & - \\
$C_{D}$ & Drag Co-efficient & 0.022 \\
$A_{r}$ & Rotor disc area & $0.083 \mathrm{~m}^{2}$ \\
$\Delta$ & Profile drag coefficient & 0.012 \\
$s$ & Rotor solidity & 0.05 \\
\hline
\end{tabular}

\section{UAV power consumption model}
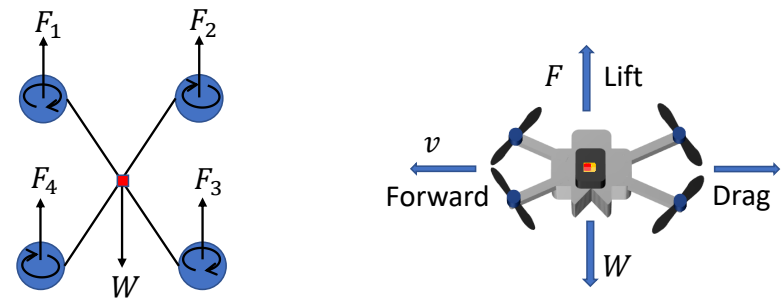

Fig. 2: Forces acting on a multi-rotor $\operatorname{UAV}\left(N_{R}=4\right)$.

The UAV carrying the radio access node ascends vertically to a HP, hovers there, and then moves horizontally from one HP to another. In this section, we provide the general expressions to calculate the total power consumed by a UAV during its hovering, horizontal and vertical movements. The definitions and values of all the variables used in this section are given in Table I. From the free-body diagram, as shown in Fig. 2, of the considered multi-rotor UAV,

$$
\sum_{n=1}^{N_{\mathrm{R}}} F_{n}-W=0
$$

The total power consumed by the UAV during its horizontal flying from one HP to another, as shown in Fig. 1, is derived using the axial momentum theory [17], [30]. Applying [10] in (67) of [17], the power required for forward flight, by assuming identical rotors $\left(F_{n}=F \forall n\right)$, is given by,

$$
\begin{aligned}
P_{\text {hfly }}(v) & =\underbrace{N_{\mathrm{R}} P_{\mathrm{b}}\left(1+\frac{3 v^{2}}{v_{\text {tip }}^{2}}\right)}_{P_{\text {blade }}}+\underbrace{\frac{1}{2} C_{D} A_{\mathrm{f}} \rho\left(h_{\mathrm{a}}\right) v^{3}}_{P_{\text {fuselage }}} \\
& +\underbrace{W\left(\sqrt{\frac{W^{2}}{4 N_{\mathrm{R}}^{2} \rho^{2}\left(h_{\mathrm{a}}\right) A_{\mathrm{r}}^{2}}+\frac{v^{4}}{4}}-\frac{v^{2}}{2}\right)^{1 / 2}}_{P_{\text {induce }}},
\end{aligned}
$$

where $\quad P_{\mathrm{b}}=\frac{\Delta}{8} \rho\left(h_{\mathrm{a}}\right) s A_{\mathrm{r}} v_{\text {tip }}^{3}, \quad \rho\left(h_{\mathrm{a}}\right) \quad=\quad(1-$ $\left.2.2558 .10^{-5} h_{\mathrm{a}}\right)^{4.2577}$. $P_{\text {blade }}$ and $P_{\text {fuselage }}$ are the powers required to overcome the profile drag forces of the rotor blades and the fuselage of the aerial vehicle that oppose its forward movement, respectively, while $P_{\text {induce }}$ represents the

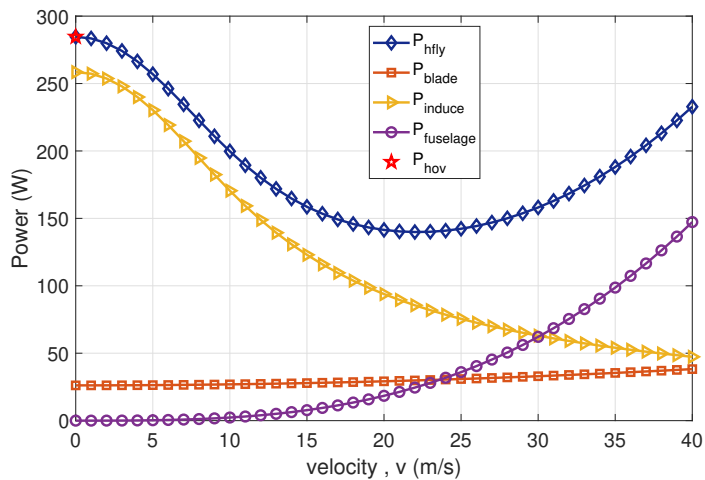

Fig. 3: UAV Power consumption components.

power required to lift the payload. The hovering power is obtained by substituting $v=0$ in (11):

$$
P_{\text {hov }}\left(h_{\mathrm{a}}\right)=N_{\mathrm{R}} P_{\mathrm{b}}+\frac{W^{3 / 2}}{\sqrt{2 N_{\mathrm{R}} \rho\left(h_{\mathrm{a}}\right) A_{\mathrm{r}}}} .
$$

Using (12.35) of [30], the power required by the aerial vehicle to climb vertically with a rate $v_{c} \mathrm{~m} / \mathrm{s}$ is expressed as,

$$
P_{\text {vfly }}=\frac{W}{2}\left(v_{\mathrm{c}}+\sqrt{v_{\mathrm{c}}^{2}+\frac{2 W}{N_{\mathrm{R}} \rho\left(h_{\mathrm{a}}\right) A_{\mathrm{r}}}}\right)+N_{\mathrm{R}} P_{\mathrm{b}} .
$$

The effect of reduced air density at higher altitudes, which demands additional force to the propeller of the UAV, is captured by modeling the hovering power as an increasing function of the altitude, as reported in (12). It is known from (11) that the drag forces are increasing functions of $v$, and are the power components that oppose them. On the other hand, the $P_{\text {induce }}$ is a decreasing function of $v$; hence, there exists an optimal velocity that minimizes $P_{\text {hfly }}(v)$, as shown in Fig. 3

\section{ENERGY EFFICIENT HOVERING POINTS}

In this section we find the energy-efficient 3-D HPs of the AAP in the given geographical area which maximize the uplink and the dowlink GEE subject to minimum Qualityof-Service (QoS), altitude, and power constraints. The corresponding downlink GEE maximization problem is formulated as follows:

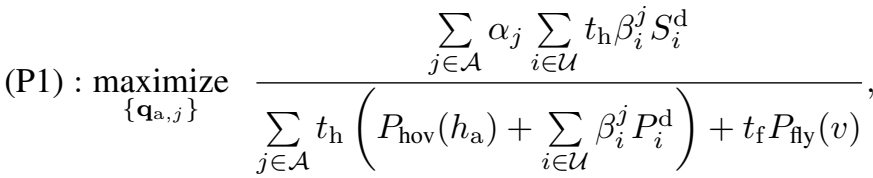

$$
\begin{aligned}
& \text { s.t. } \quad h_{\min } \leq q_{v, j}^{\mathrm{a}} \leq h_{\max }, \forall j \in \mathcal{A} \text {, } \\
& S_{i}^{\mathrm{d}} \geq S_{\mathrm{o}}^{\mathrm{d}} \forall i \in \mathcal{U}, \\
& \sum_{i \in \mathcal{U}} \beta_{i}^{j} P_{i}^{\mathrm{d}} \leq P_{\text {max }}^{\mathrm{d}} \forall j \in \mathcal{A}, \\
& \sum_{j \in \mathcal{A}} \beta_{i}^{j}=1 \quad \forall i \in \mathcal{U}, \\
& \left\|\mathbf{q}_{\mathrm{h}, j}^{\mathrm{a}}-\beta_{i}^{j} \mathbf{q}_{h, i}^{\mathrm{ue}}\right\| \leq\left|q_{\mathrm{v}, j}^{\mathrm{a}}\right| \tan \theta \forall j \in \mathcal{A} .
\end{aligned}
$$

The numerator of (P1) is the sum of the data rate of all the UEs in the given geographical area. The binary indicator variable $\alpha_{j}$ is equal to 1 if the AAP is hovering at the $j^{\text {th }} \mathrm{HP}$ with 
coordinates $\left(\mathbf{q}_{h, j}^{\mathrm{a}}, q_{\mathrm{v}, j}^{\mathrm{a}}\right)$, or to 0 otherwise; $\beta_{i}^{j}$ is equal to 1 if the $i^{\text {th }}$ UE located at $\left(\mathbf{q}_{\mathrm{h}, i}^{\mathrm{ue}}, 0\right)$ is covered by the AAP while hovering at the $j^{\text {th }}$ HP; $\mathcal{A}$ and $\mathcal{U}$ are the sets of the HPs and UEs, respectively. (14), (15), and (16) are the altitude, QoS and power constraints; $h_{\min }$ and $h_{\max }$ are the minimum and maximum permitted AAP altitudes. (17) expresses that each UE should be covered by the AAP while hovering at any of the $|\mathcal{A}|$ HPs. $(18)$ is the coverage region constraint that couples the horizontal and vertical coordinates of a HP. The denominator of the ratio in (P1) is the sum of the hovering and the flying energy consumption, with $t_{\mathrm{h}}$ and $t_{\mathrm{f}}$ being the hovering time at a HP and the total flying time respectively. (P1) has the form of a mixed-integer non-linear fractional problem (MINLFP), which is exponentially complex to solve due to the constraint (18). Considering the uplink GEE to be jointly maximized with the downlink GEE would enhance the complexity further; hence we decouple the AAP placement problem in the vertical and horizontal dimensions [5], [19].

\section{A. Optimal vertical coordinates of the hovering points}

The energy-efficient hovering altitude of the AAP considering both the uplink and downlink communication between the AAP and the UEs is analytically derived in this section. Here we consider the instant in which the AAP is hovering at HP1 as shown in Fig. 11 with 3-D coordinates $\left(\mathbf{q}_{\mathrm{h}, 1}^{\mathrm{a}}, q_{\mathrm{v}, 1}^{\mathrm{a}}\right)=\left(0,0, h_{\mathrm{a}}\right)$. The problem that finds the optimal altitude that maximizes both the uplink and downlink GEE of the considered aerial communication network subject to the altitude, power, and QoS constraints can be formulated as,

$$
\begin{aligned}
\text { (P2) : } & \underset{h_{\mathrm{a}}}{\operatorname{maximize}} \quad\left(\mathrm{GEE}_{\mathrm{d}}, \mathrm{GEE}_{\mathrm{u}}\right), \\
\text { s.t. } & h_{\min } \leq h_{\mathrm{a}} \leq h_{\max }, \\
& S_{i}^{\mathrm{d}} \geq S_{\mathrm{o}}^{\mathrm{d}} ; S_{i}^{\mathrm{u}} \geq S_{\mathrm{o}}^{\mathrm{u}} \quad \forall i: r_{i} \leq R_{\mathrm{a}}, \\
& P_{t}^{\mathrm{d}} \leq P_{\max }^{\mathrm{d}}, \\
& P_{i}^{\mathrm{u}} \leq P_{\max }^{\mathrm{u}} \quad \forall i: r_{i} \leq R_{\mathrm{a}},
\end{aligned}
$$

where, $\mathrm{GEE}_{\mathrm{d}}=\bar{S}_{\mathrm{d}} / P_{\mathrm{t}}^{\mathrm{d}}$ and $\mathrm{GEE}_{\mathrm{u}}=\bar{S}_{\mathrm{u}} / P_{\mathrm{t}}^{\mathrm{u}}$ are the uplink and downlink GEE of the system, respectively, with $P_{\mathrm{t}}^{\mathrm{d}}, P_{\mathrm{t}}^{\mathrm{u}}$ being the respective total downlink and uplink AAP power consumption while hovering, expressed by (23) and (24):

$$
\begin{aligned}
P_{\mathrm{t}}^{\mathrm{d}} & =\underbrace{\pi \lambda_{\mathrm{u}} h_{\mathrm{a}}^{2} \tan ^{2} \theta P_{i}^{\mathrm{d}}}_{P_{\mathrm{com}}^{\mathrm{d}}}+P_{\mathrm{hov}}\left(h_{\mathrm{a}}\right), \\
P_{\mathrm{t}}^{\mathrm{u}} & =\underbrace{\frac{2 \pi \lambda_{\mathrm{u}} P_{\mathrm{a}} L_{\mathrm{m}}(\theta) \tan ^{2} \theta h_{\mathrm{a}}^{4}\left[\tan ^{2} \theta+2\right]}{4 g_{\mathrm{o}}}}_{P_{\text {com }}^{\mathrm{u}}}+P_{\mathrm{hov}}\left(h_{\mathrm{a}}\right),
\end{aligned}
$$

where $P_{\mathrm{com}}^{\mathrm{d}}$ and $P_{\mathrm{com}}^{\mathrm{u}}$ are the respective total communicationrelated power consumption values; $P_{\mathrm{com}}^{\mathrm{u}}$ of (24), is obtained by averaging the power profile over a uniform distribution of density $\lambda_{\mathrm{u}}$, with the approximation $L_{\mathrm{m}}\left(\phi_{i}\right) \approx L_{\mathrm{m}}\left(\phi_{\text {edge }}\right) \forall i$ : $r_{i} \leq R_{\mathrm{a}}$ [19]. (19) and (20) are the altitude and QoS constraints with $S_{\mathrm{o}}^{\mathrm{d}}, S_{\mathrm{o}}^{\mathrm{u}}$ being the respective minimum downlink and uplink data rates in bps; 21 and 22 are the total downlink and individual UE uplink power constraints. Considering the edge UE $\left(r_{i}=R_{\mathrm{a}}\right)$, the uplink QoS constraint becomes $P_{\mathrm{a}}=\delta_{\mathrm{u}} \Gamma_{i} \sigma^{2} / G_{\mathrm{a}}$; furthermore, the downlink QoS constraint, 20), and the power constraints, (21) and (22), can be equivalently represented as the altitude constraints in 25, (26), and 27):

$$
\begin{aligned}
& h_{\mathrm{a}} \leq h_{\max , \delta_{\mathrm{d}}}^{\mathrm{d}}=\cos \theta \sqrt{\frac{G^{\prime}}{\delta_{\mathrm{d}}}}, \\
& h_{\mathrm{a}} \leq h_{\mathrm{max}, P}^{\mathrm{d}}=\sqrt{\frac{P_{\mathrm{max}}^{\mathrm{d}}-P_{\mathrm{hov}}}{\pi \lambda_{\mathrm{u}} \tan ^{2} \theta P_{i}^{\mathrm{d}}}}, \\
& h_{\mathrm{a}} \leq h_{\max , P}^{\mathrm{u}}=\cos \theta \sqrt{\frac{g_{\mathrm{o}} P_{\max }^{\mathrm{u}}}{P_{\mathrm{a}} L_{\mathrm{m}}(\theta)}},
\end{aligned}
$$

where $\delta_{\mathrm{d}}=2^{S_{\mathrm{o}}^{\mathrm{d}} / B}-1$ and $\delta_{\mathrm{u}}=2^{S_{\mathrm{o}}^{\mathrm{u}} / B}-1$. Hence, (P2) can be reformulated as

$$
\begin{aligned}
& \text { (P2.1) }: \underset{h_{\mathrm{a}}}{\operatorname{maximize}}\left(\mathrm{GEE}_{\mathrm{d}}, \mathrm{GEE}_{\mathrm{u}}\right), \\
& \text { s.t. } h_{\min } \leq h_{\mathrm{a}} \leq \min \left\{h_{\max }, h_{\max , \delta_{\mathrm{d}}}^{\mathrm{d}}, h_{\max , \mathrm{P}}^{\mathrm{d}}, h_{\max , \mathrm{P}}^{\mathrm{u}}\right\}
\end{aligned}
$$

Proposition 2: For given $\theta, S_{\mathrm{o}}^{\mathrm{d}}$, and $S_{\mathrm{o}}^{\mathrm{u}}$ values;

Statement A: The GEE of the system considering the downlink communication between the AAP and the ground UEs lying in the AAP coverage region is an increasing function of the hovering altitude.

Statement B: The GEE of the system considering the uplink communication between the AAP and the ground UEs lying in the AAP coverage region is an increasing function of the hovering altitude.

Proof: The derivative of the numerator and the denominator of the downlink GEE with respect to $h_{\mathrm{a}}$ are given by:

$$
\begin{gathered}
\frac{\bar{S}_{\mathrm{d}}^{\prime}}{2 h_{\mathrm{a}} B^{\prime}}=\sec ^{2} \theta \log \left(\frac{G^{\prime}+h_{\mathrm{a}}^{2} \sec ^{2} \theta}{h_{\mathrm{a}}^{2} \sec ^{2} \theta}\right)-\log \left(\frac{G^{\prime}+h_{\mathrm{a}}^{2}}{h_{\mathrm{a}}^{2}}\right), \\
P_{t}^{\prime \mathrm{d}}=2 \pi \lambda_{\mathrm{u}} h_{\mathrm{a}} \tan ^{2} \theta P_{i}^{\mathrm{d}}+P_{\mathrm{hov}}^{\prime}\left(h_{\mathrm{a}}\right),
\end{gathered}
$$

where $P_{\text {hov }}^{\prime}\left(h_{\mathrm{a}}\right)=N_{\mathrm{R}} \frac{\Delta}{8} \rho^{\prime}\left(h_{\mathrm{a}}\right) s A_{\mathrm{r}} v_{\text {tip }}^{3}-\frac{W^{3 / 2} \rho^{\prime}\left(h_{\mathrm{a}}\right)}{\sqrt{8 N_{\mathrm{R}} A_{\mathrm{r}}} \rho^{3 / 2}\left(h_{\mathrm{a}}\right)}$ with $\rho^{\prime}\left(h_{\mathrm{a}}\right)=-9 \cdot 6 \cdot 10^{-5}\left(1-2.2558 \cdot 10^{-5} h_{\mathrm{a}}\right)^{3.2577}$. Hence the derivative of the downlink GEE with respect to $h_{\mathrm{a}}$ is

$$
\begin{aligned}
\left(P_{\mathrm{t}}^{\mathrm{d}}\right)^{2}\left(\frac{\bar{S}_{\mathrm{d}}}{P_{\mathrm{t}}^{\mathrm{d}}}\right)^{\prime} & =\bar{S}_{\mathrm{d}}^{\prime} P_{\mathrm{t}}^{\mathrm{d}}-P_{\mathrm{t}}^{\prime} \mathrm{d} \bar{S}_{\mathrm{d}}, \\
& >0 \quad \forall h \in\left\{h_{\min }, h_{\max }\right\} .
\end{aligned}
$$

The increasing nature of the uplink GEE with respect to $h_{\mathrm{a}}$ can be proved using similar steps used in the proof of Statement A with the following numerator and denominator derivatives:

$$
\begin{gathered}
\bar{S}_{\mathrm{u}}^{\prime}=2 B \pi \lambda_{\mathrm{u}} \tan ^{2} \theta S_{i}^{\mathrm{u}} h_{\mathrm{a}} \\
P_{t}^{\prime} \mathrm{u}=\frac{2 \pi \lambda_{\mathrm{u}} P_{\mathrm{a}} L_{\mathrm{m}}(\theta) \tan ^{2} \theta\left[\tan ^{2} \theta+2\right] h_{\mathrm{a}}^{3}}{g_{\mathrm{o}}}+P_{\mathrm{hov}}^{\prime}\left(h_{\mathrm{a}}\right) \text {. }
\end{gathered}
$$

Thus, by Proposition 1, the globally energy-efficient hovering altitude for the AAP that maximizes both the uplink and downlink GEE (the objective function of (P2) ) is $h_{\mathrm{opt}}=$ $\min \left\{h_{\max }, h_{\text {max }, \delta_{\mathrm{d}}}^{\mathrm{d}}, h_{\text {max }, \mathrm{P}}^{\mathrm{d}}, h_{\text {max }, \mathrm{P}}^{\mathrm{u}}\right\}$. 
1) Optimal Beamwidth: For a given altitude, both the uplink and downlink GEE of the system vary with the beamwidth of the antenna at the UAV. As the beamwidth increases, the coverage area increases, the effective antenna gain given by (1) decreases, and the additional path loss factor, $L_{\mathrm{m}}(\theta)$, increases. Here we find the optimal beamwidth that jointly maximizes the uplink and downlink GEE of the system.

Proposition 3: For a given QoS constraint, the uplink-GEE is an increasing function of the beamwidth.

Proof: The derivative of the uplink sum rate is given by:

$$
\frac{d \bar{S}_{\mathrm{u}}}{d \theta}=2 B \pi \lambda_{\mathrm{u}} h_{\mathrm{a}}^{2} \tan \theta \sec ^{2} \theta \log _{2}\left(1+\delta_{\mathrm{u}}\right)>0 .
$$

Since $P_{\mathrm{com}}^{\mathrm{u}}<<P_{\text {hov }}\left(h_{\mathrm{a}}\right), P_{\mathrm{t}}^{\mathrm{u}} \approx P_{\text {hov }}$ makes the denominator of the uplink GEE an independent function of $\theta$. Hence, the optimal beamwidth that maximizes the uplink-GEE of the considered system is $\theta_{\mathrm{opt}}^{\mathrm{u}}=\theta_{\max }$.

Using (7) and $P_{\mathrm{t}}^{\mathrm{u}} \approx P_{\text {hov }}$, it can be pointed out that the downlink GEE is neither an increasing function nor a decreasing function of $\theta$. Hence, for a given hovering altitude, the optimal $\theta$ that maximizes the downlink GEE can be determined using the ternary search method, as detailed in Algorithm 1. Therefore, the antenna beamwidth that maximizes

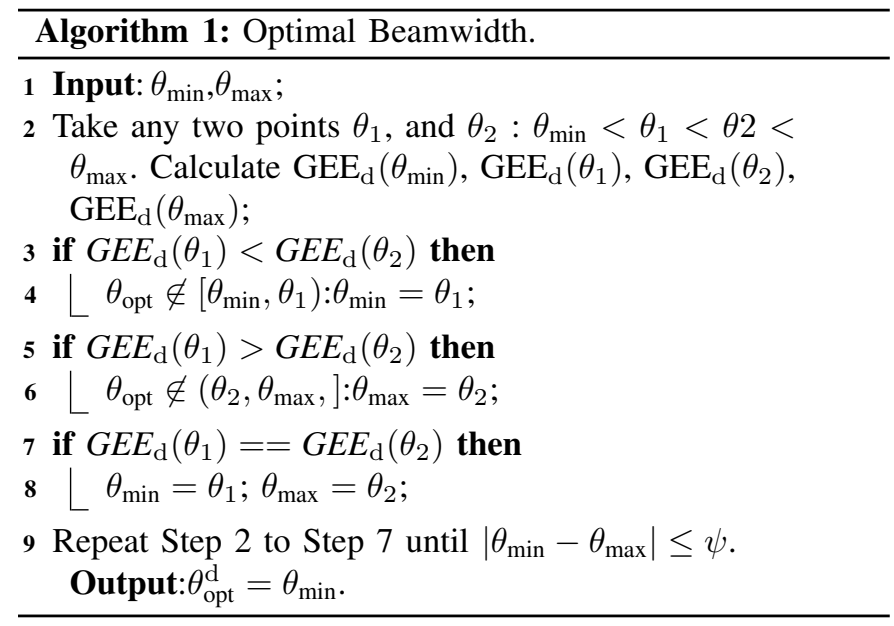

both the uplink and the downlink GEE is $\theta_{\mathrm{opt}}=\min \left(\theta_{\mathrm{opt}}^{\mathrm{u}}, \theta_{\mathrm{opt}}^{\mathrm{d}}\right)$. Hence, the corresponding radius of the coverage region is given by $R_{\mathrm{opt}}=h_{\mathrm{opt}} \tan \theta_{\mathrm{opt}}$.

\section{B. Optimal horizontal coordinates of the HPs}

In this section, we determine the horizontal coordinates of the optimal HPs for the AAP $\left(\left\{\mathbf{q}_{\mathrm{h}, j}^{\mathrm{a}}\right\}\right)$ so that all the UEs in the given geographical region will be covered by the AAP once every $T_{\mathrm{n}} \mathrm{s}$. The vertical coordinate of all the HPs will be equal to the optimal altitude determined from Section III-A. i.e. $q_{\mathrm{v}, j}^{\mathrm{a}}=h_{\mathrm{opt}} \forall j \in \mathcal{A}$. Since the coverage region of the considered AAP is circular in shape, a full coverage of the given area is only achievable through the controlled overlapping among the coverage regions centered at $\left\{\left(\mathbf{q}_{\mathrm{h}, j}^{\mathrm{a}}, 0\right)\right\}$. The two main related challenges are finding the minimum number of HPs and their horizontal coordinates so that the given area is fully covered. We propose a MCP algorithm for doing so, using the circle packing theory. With the optimal coverage radius $R_{\text {opt }}$ from Section III-A the problem takes the form of a circle packing problem, in which the given circle of radius $R$ needs to be covered by smaller circles of radius $R_{\text {opt }}$. A regular-pentagon-based 5-circle packing pattern is considered in the proposed novel MCP algorithm. As shown in Fig. 7. the 5-circle packing covers a circle of radius $\Lambda R_{\text {opt }}$ by placing five equi-radius smaller circles of radius $R_{\text {opt }}$ about the center of the larger circle, where $\Lambda=1.618$ is the golden ratio [25]. The smaller circles are placed according to the solution of the 5-disks problem, which guarantees the least overlapping between the adjacent circles [25]. The centers of the smaller circles arranged this way form the vertices of a regular pentagon of side length $2 \sin \left(36^{\circ}\right) \Lambda R_{\text {opt }}$, with vertices located at $\left\{\left(x_{k}^{l}, y_{k}^{l}\right)\right\}$ where,

$$
\begin{aligned}
& x_{k}^{l}=x^{l-1}+R_{l} \cos \left(\frac{2 \pi k}{5}\right) \quad \text { for } k \in\{0,1, \ldots 4\}, \\
& y_{k}^{l}=y^{l-1}+R_{l} \sin \left(\frac{2 \pi k}{5}\right) \quad \text { for } k \in\{0,1, \ldots 4\},
\end{aligned}
$$

with $\left(x^{l-1}, y^{l-1}\right)$ as the center of the pentagon and $R_{l}=R_{\text {opt }}$. Thus, to cover a circular region with $R>\Lambda R_{\text {opt }}$, we initially place this 5-circle pattern in multiple levels as shown in Fig. 4. and then the radius of each circle is adjusted according to the farthest UE's position in the circle.

Proposition 2: Using the multi-level 5 circle packing method, the maximum number of HPs required to cover a given circular region of radius $R$ by a fly-hover communicating AAP with a coverage radius of $R_{\text {opt }}$ is given by $5^{M_{p}}$, where $M_{p}=\left\lceil\frac{1}{\log _{2}(\Lambda)} \log _{2}\left(\frac{R}{R_{\mathrm{opt}}}\right)\right\rceil$.

Proof: According to the multi-level 5 circle packing method, the radius of the circle to be covered in each level is $1 / \Lambda$ times the radius of the circle of the previous level. This multi-level packing continues until the radius of the circle to be covered is less than or equal to the coverage radius of the $\mathrm{AAP}$, giving the inequality:

$$
R \frac{1}{\Lambda^{M_{p}}} \leq R_{\mathrm{opt}}
$$

Thus, the total number of levels required is given by:

$$
M_{\mathrm{p}}=\left\lceil\frac{1}{\log _{2}(\Lambda)} \log _{2}\left(\frac{R}{R_{\mathrm{opt}}}\right)\right\rceil .
$$

Hence the maximum number of smaller circles (HPs) of radius $R_{\text {opt }}$ required to cover the given geographical region of radius $R$ by the 5 -circle multi-level packing is $5^{M_{p}}$.

1) MCP Algorithm: The MCP algorithm given in Algorithm 2 can be grouped into two parts: in the first part (line 1 to line 10), the locations of the HPs are determined using the multi-level 5-circle packing method; in the second part (lines 11 to 16), the beamwidth of the antenna at each HP is adjusted based on the UE positions in the coverage area so that the constraints (17) and (18) are satisfied. Consider $R=733 \mathrm{~m}, h_{\mathrm{opt}}=102 \mathrm{~m}$ and $\theta=70^{\circ}$; using (38), the total number of circle packing levels required to cover the given area is 2. As shown in Fig. 4, in the first level of placement $(l=1)$, the location of the centers of 5 smaller circles of 


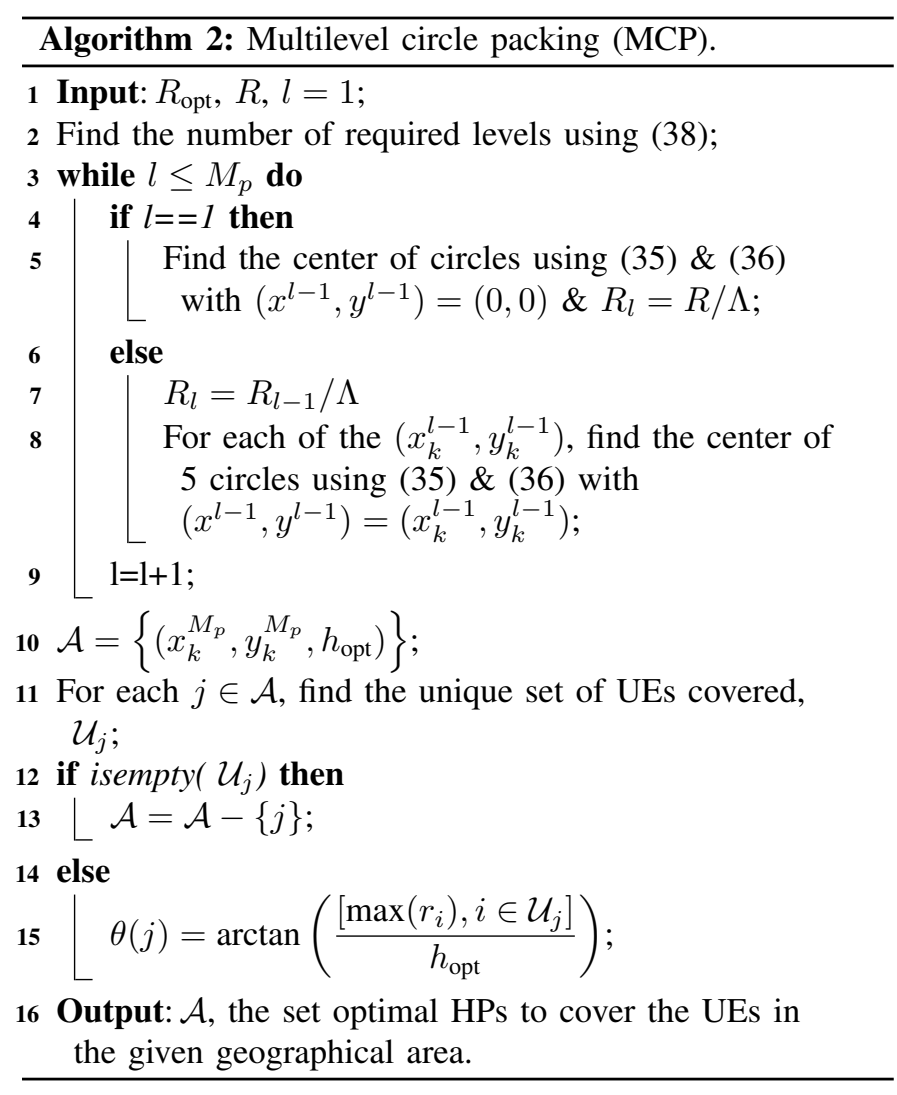

radius $R_{1}=R / \Lambda$ are determined using step 5 of the $\mathrm{MCP}$ algorithm (A,B,C,D,E of Fig. 4). In the next level, step 8 is used to determine the location of the centers of 5 smaller circles of radius $R_{2}=R_{1} / \Lambda$ that cover the circle of radius $R_{1}$. This step is repeated for each of the 5 determined centers from the first level. The centers of the smaller circles of radius $R_{2}$ are the HPs that cover the given geographical area. As seen in Fig. 4, the determined HPs lie on the vertices of 5 smaller pentagons with circumcenters A, B, C, D, E. For any given $R$, this multi-level circle packing continues until the radius of the circle to be covered is less than, or equal to, the coverage radius of the AAP, or until $l=M_{p}$. In the second part, redundant coverage of the same UE by multiple HPs and the HPs without any UEs in the coverage region are removed. The coverage radius associated with each HP is readjusted as the radial distance of the farthest UE lying in the respective coverage areas by adjusting the antenna beamwidth using step 15. It should be noted that, with given $R$ and $R_{\text {opt }}$, the first part takes a total running time of $O\left[\log _{2}\left(R / R_{\text {opt }}\right)\right]$ to find the initial set of HPs, whereas the second part needs to perform a total of $\sum_{f=0}^{|\mathcal{A}|-1}(|\mathcal{A}|-f)(|\mathcal{A}|-f-1)$ pair checks to remove the redundant UE coverage where $|\mathcal{A}|=5^{M_{\mathrm{p}}}$. Hence the complexity of the proposed MCP algorithm is, at worst $O\left[\log _{2}\left(R / R_{\mathrm{opt}}\right)+5^{\omega \log _{2}\left(R / R_{\mathrm{opt}}\right)}\right]$, a polynomial quantity of the input parameters $R$ and $R_{\text {opt }}$; where $\omega=2 / \log _{2} \Lambda$. It should be noted that this algorithm could maximize different objective functions in which the coverage radius of the AAP will be determined by the very objective function. Also, it can be adapted to different packing patterns by replacing the equations in step 5 and 8 .

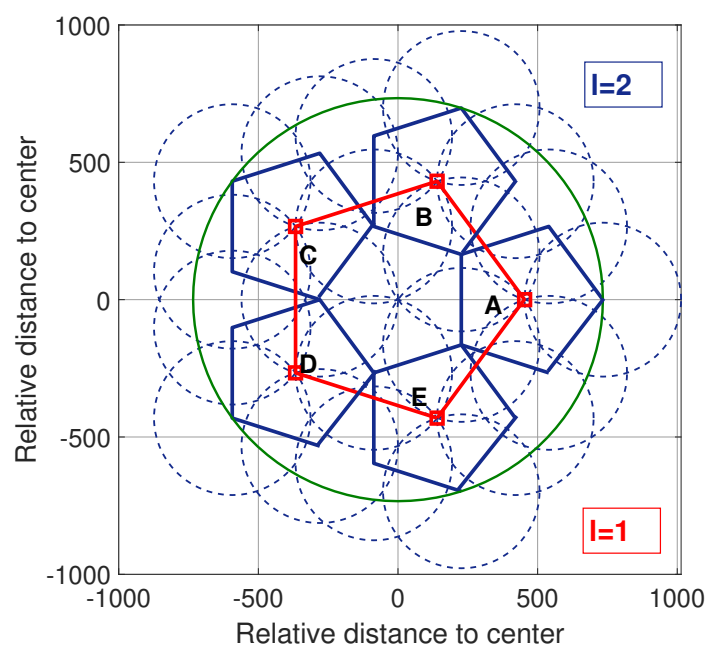

Fig. 4: AAP coverage circles positioned using the first part of the MCP algorithm for $R=733 \mathrm{~m}$.

\section{ECONOMIC ANALYSIS AND COST OPTIMISATION}

In this section, we describe the cost optimization method developed for an aerial network and the parameters it uses. The total aerial network deployment cost consists of two parts: a) the cost of purchasing the required number of UAVs and recharging stations, called the capital cost; b) the maintenance and replacement cost associated with the long-term operation of the network, represented by the operational cost. The UAV is equipped with two TB47D LiPo Battery, for a total capacity $E_{\text {batt }}=199.8 \mathrm{Wh}$. Our objective is to minimize the network deployment cost by optimally planning the operational parameters.

The financial analysis is based on the annualized cost of the system (ACS), defined as the sum of the annualized capital, maintenance, and replacement costs [27]:

$$
\mathrm{ACS}=C_{\text {cap,a }}+C_{\text {main,a }}+C_{\text {rep,a }} .
$$

The annualization is needed in order to report different expenses to the same time interval (year of reference) so that expenses happening at different times can be compared. $C_{\text {cap,a }}$ is given by (40), where the capital cost is multiplied by the capital recovery factor (CRF), i.e., the portion of capital that is paid back every year to the financier:

$$
C_{\text {cap }, \mathrm{a}}=\underbrace{\left(n_{\mathrm{u}} C_{\mathrm{u}}\right)+\left(n_{\mathrm{s}} C_{\mathrm{s}}\right)}_{C_{\text {cap }}} \cdot \underbrace{\frac{I(1+I)^{\mathrm{lt}}}{(1+I)^{\mathrm{tt}}-1}}_{\text {CRF }},
$$

with $C_{\mathrm{u}}$ and $C_{\mathrm{s}}$ being the purchasing cost per unit of, respectively, UAV and charging station. The financing method is based on debt, with a duration of 15 years (lifetime of the system), a nominal interest rate of $4 \%[28]$ and an inflation rate of $2 \%$, meaning a real interest rate $I \approx 2 \% . n_{\mathrm{s}}$ is the number of charging stations and $n_{\mathrm{u}}$, the number of UAVs in the system (both active and idle), determined as:

$$
n_{\mathrm{u}}=\left(\frac{t_{\text {dead }}}{t_{\text {active }}}+1\right)\left\lceil\min \left(|\mathcal{A}|, \frac{t_{\text {lap }}(v)}{T_{\mathrm{n}}}\right)\right\rceil,
$$


where $t_{\text {dead }}$ represents all the dead time an AAP spends recharging and ascending/descending, as expressed in (42); $t_{\text {active }}$ is the total active time of a UAV; $t_{\text {lap }}(v)$ is the time needed to complete a lap, stopping at all the HPs. Given 400 and (41), for a low $T_{\mathrm{n}}$ value, the number of UAVs, and thus the capital cost, is determined by the number of HPs $(|\mathcal{A}|)$. Hence, by finding the minimum number of HPs required to cover all the UEs (Algorithm 2), we minimize the capital cost. The $t_{\text {dead }}$ value is calculated assuming a maximum charging power $P_{\text {ch,max }}$, a DOD that can go from $5 \%$ to $95 \%$, and a fixed charging efficiency $\eta_{\mathrm{ch}}$, as follows:

$$
t_{\text {dead }}=\frac{E_{\text {batt }} \mathrm{DOD}}{P_{\mathrm{ch}, \max }} \eta_{\mathrm{ch}}+t_{\mathrm{ex}},
$$

where $t_{\mathrm{ex}}$ is the unproductive time spent by the UAV climbing to take its position in the network and descending for recharge. The cost of the electricity consumed to recharge the batteries is given by the product of the energy provided by the charging station and the cost of electricity in the network deployment region (in the $\mathrm{UK}, C_{\mathrm{el}}=0.14 £ / \mathrm{kWh}$ ):

$$
C_{\mathrm{ch}}=\frac{t_{\mathrm{miss}}}{t_{\mathrm{active}}} C_{\mathrm{el}} \frac{E_{\mathrm{UAV}}\left(v, h_{\mathrm{a}}\right)}{\eta_{\mathrm{ch}}},
$$

where $E_{\mathrm{UAV}}\left(v, h_{\mathrm{a}}\right)=|\mathcal{A}| t_{\mathrm{h}} P_{\text {hov }}\left(h_{\mathrm{a}}\right)+t_{\mathrm{f}} P_{\text {hfly }}(v)+t_{\mathrm{ex}} P_{\text {vfly }}$ and $t_{\text {miss }}=2 \mathrm{hr}$ is the duration of an entire mission. $C_{\text {main,a }}$ represents both the yearly maintenance cost (assumed equal to $1 \%$ of the capital cost) and the cost of a recharge, adjusted for inflation $f$ after $n$ years using $C_{\text {main,a }}=\left(C_{\mathrm{ch}}+\right.$ $\left.1 \% C_{\text {cap }}\right)(1+f)^{n}$. The last element of 399 is represented by the annualized cost of battery replacement, $C_{\text {rep,a }}$, calculated using,

$$
C_{\text {rep,a }}=C_{\text {bat }} \frac{I}{(1+I)^{l_{\text {bat }}}-1},
$$

with $l_{\text {bat }}$ being the lifetime of the battery and $C_{\text {bat }}$ its replacement cost (£155). For simplicity, the lifetime of the battery is assumed to depend solely on the DOD, and it is simulated using the model presented in [29], reported in [45]:

$$
n_{\mathrm{c}}=8.131 e^{4(-0.03809 \mathrm{DOD})}+2.151 e^{-8(0.2433 \mathrm{DOD})} \text {. }
$$

This returns the number of cycles $n_{\mathrm{c}}$ a $\mathrm{LiFePO}_{4}$ battery can withstand at a certain DOD, which is then converted into years using the mission duration and its frequency.

$C_{\text {cap,a }}, C_{\text {main,a }}$, and $C_{\text {rep,a }}$ can be minimized by suitably selecting the DOD value and the velocity of the UAV. $C_{\text {cap,a }}$ can be minimized by increasing the DOD, since this increases $t_{\text {active }}$. It can be noted that $t_{\text {dead }}$ also grows with the DOD, but at a slower rate, due to the attenuation effect of $t_{\mathrm{ex}}$. However, (41) is a step function, so the variations in these parameters do not always affect $n_{\mathrm{u}}$. By (43), $C_{\text {main,a }}$ can be reduced by increasing $t_{\text {active }}$ through the selection of a high DOD value. On the contrary, since the lifetime of the battery is increased by a low DOD value, the battery replacement $\operatorname{cost} C_{\text {rep,a }}$ can only be minimized by selecting a low DOD value. For a fixed network delay tolerance, $T_{\mathrm{n}}$, the UAV velocity affects both the power consumption and the number of UAVs: though the minimum power consumption is reached at an average speed of 20 $\mathrm{m} / \mathrm{s}$ (Fig. 3), the minimum cost is expected to be achieved
TABLE II: Simulation Parameters.

\begin{tabular}{|l|l|l|l|}
\hline Parameter & Value & Parameter & Value \\
\hline$g_{\mathrm{o}}$ & $1.42 \times 10^{-4}$ & $\mathrm{~B}$ & $10^{6} \mathrm{~Hz}$ \\
\hline$h_{\max }$ & $120 \mathrm{~m}$ & $\lambda_{\mathrm{u}}$ & $0.001 \mathrm{UEs} / m^{2}$ \\
\hline$\delta_{\mathrm{d}}, \delta_{\mathrm{u}}$ & $9 \mathrm{~dB}$ & $h_{\min }$ & $10 \mathrm{~m}$ \\
\hline$\Gamma_{i} \forall i \in \mathcal{U}$ & 1.2 & $P_{\max }^{\mathrm{d}}$ & $500 \mathrm{~W}$ \\
\hline$P_{\max }^{\mathrm{u}}$ & $1 \mathrm{~W}$ & $C_{\mathrm{u}}$ & $£ 2000$ \\
\hline$C_{\mathrm{s}}$ & $£ 1000$ & $C_{\mathrm{bat}}$ & $£ 155$ \\
\hline$\eta_{\mathrm{ch}}$ & 0.95 & $P_{\mathrm{ch}, \max }$ & $180 \mathrm{~W}$ \\
\hline
\end{tabular}

when the UAV is operated at its maximum velocity because a higher velocity minimizes the capital cost by reducing the $t_{\text {lap }}(v)$ value. Considering these trade-offs, the values of DOD and horizontal flying velocity that minimize the ACS will be numerically determined in the next section.

\section{NUMERICAL RESUlTS AND DISCUSSION}

In this section, we provide the results of the numerical evaluation in support of our analysis. The considered simulation parameters are given in Table II.

\section{A. Energy efficient hovering points}

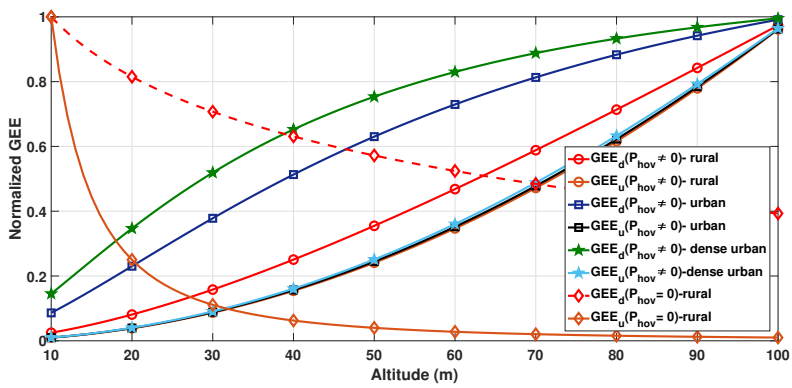

Fig. 5: GEE variation with the hovering altitude.

Fig. 5 shows the variation of the normalized GEE with the hovering altitude for both the uplink and downlink communication parts, plotted using (7), 23), 97, and (24). The $\left(a, b, \eta_{1}, \eta_{\mathrm{nl}}\right)$ parameters used for rural, urban, and dense-urban regions are, respectively, $(4.83,0.43,1.01,11.22)$, $(9.6,0.16,1.12,10),(12.08,0.11,1.2,14.12)$ [4]. As proved in Proposition 1, both the uplink and downlink GEE are increasing functions of the hovering altitude in the three geographical regions considered. As the altitude increases, the received SNR at each UE (downlink) decreases because of the increasing $d_{i}$ value. In contrast, the received SNR at the AAP from the UEs (uplink) is independent of the hovering altitude because of the uplink power control mechanism. Nevertheless, a higher hovering altitude demands the UEs to transmit more power to deliver the target power $\left(P_{\mathrm{a}}\right)$ at the AAP. However, this increase in the communication-related power consumption is negligible, if compared to the UAV hovering power $\left.\left(P_{\text {hov }}\left(h_{\mathrm{a}}\right)\right)\right)$. Both the AAP coverage area and the UAV power consumption increase with the altitude, but the effect of the larger area (higher number of covered UEs) is more relevant in both cases, which makes the downlink and uplink GEE monotonically increasing functions of the hovering altitude. It should be noted that, in both cases, if $P_{\text {hov }}\left(h_{\mathrm{a}}\right)$ is neglected, 
the increase in the communication power becomes significant and the GEE becomes in fact a decreasing function of the altitude. Thus $h_{\mathrm{opt}}\left(P_{\text {hov }}\left(h_{\mathrm{a}}\right)=0\right)=h_{\text {min }}$, which is not only energy-inefficient, but also requires more HPs to cover the given area (cost-inefficient).

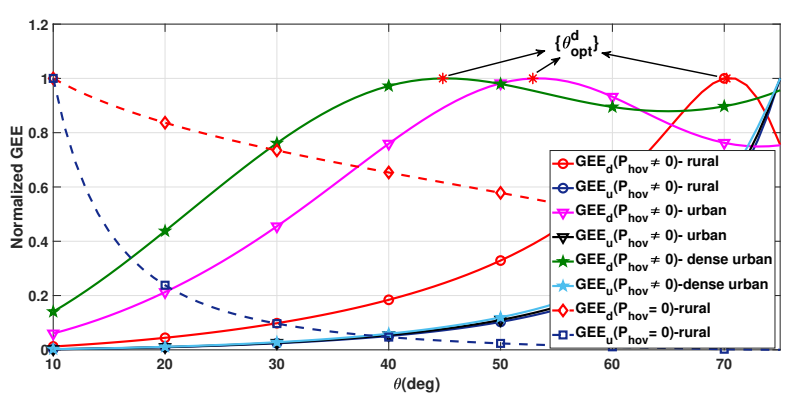

Fig. 6: GEE variation with the antenna beamwidth.

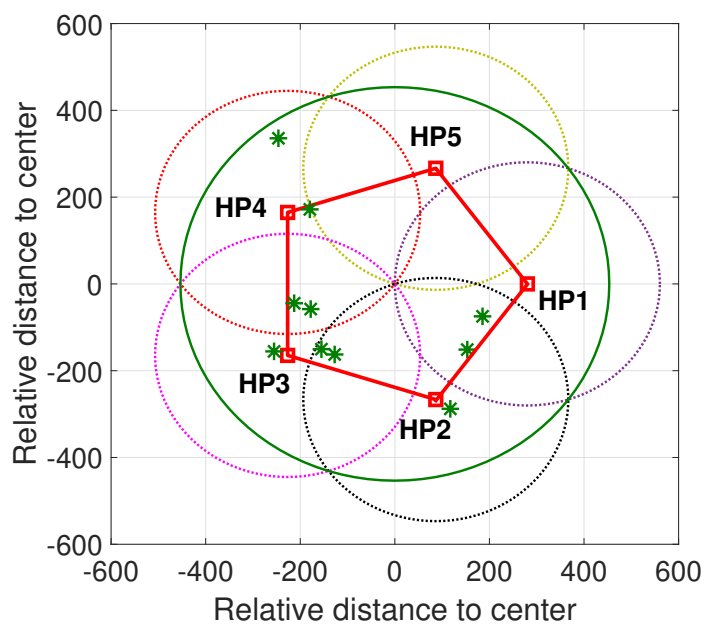

Fig. 7: AAP coverage circles positioned using the first part of the MCP algorithm for $R=453 \mathrm{~m}$.

Fig. 6 shows the variation of the GEE with half of the half-power beamwidth, $\theta$, in rural, urban, and dense-urban scenarios. As said in Section III-A1, the coverage radius is an increasing function of $\theta$; however, for a given height, Fig. 6 shows that the downlink GEE increases with $\theta$ up to $\theta_{\mathrm{opt}}^{\mathrm{d}}$ (obtained using Algorithm 1), then decreases. This is because the LoS probability is a decreasing function of $\theta$ and, after $\theta_{\mathrm{opt}}^{\mathrm{d}}$, the high path loss NLoS links become dominant; hence, the gain in sum rate achieved by a greater number of covered UEs is overcompensated by a drop in the received SNR. On the other hand, the uplink GEE is an increasing function of $\theta$ : because of the power control mechanism, the uplink sum-rate grows with the number of UEs in the coverage region, which is, in turn, an increasing function of $\theta$. However, the maximum value of $\theta$ will be decided by the $P_{\max }^{\mathrm{u}}$ of the edge UE. Furthermore, the optimal beamwidth decreases as we move from rural to dense-urban regions due to the presence of taller and more numerous buildings. In both the uplink and downlink scenarios, the plots with $P_{\text {hov }}\left(h_{\mathrm{a}}\right)=0$ are decreasing functions of $\theta$, forcing the elevation angle between the UEs and the AAP to be $0^{\circ}$. This means that, with $P_{\text {hov }}\left(h_{\mathrm{a}}\right)=0$, the AAP should hover directly above the UE locations to maximize the GEE.
This would be a sub-optimal result, as the number of HPs would be equal to that of UEs.

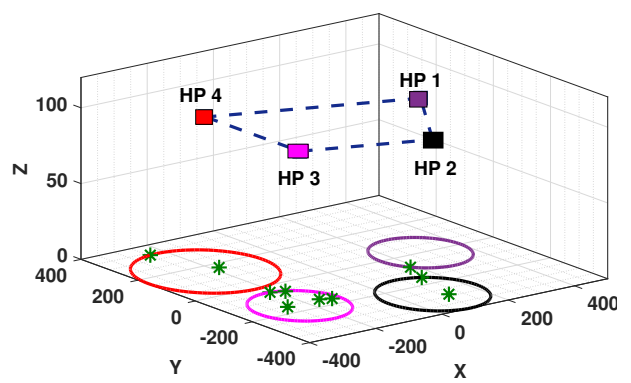

Fig. 8: 3-D HPs using the MCP algorithm for $R=453 \mathrm{~m}$.

Fig. 8 shows the final HP locations in the given geographical area of $R=453 \mathrm{~m}$, determined using the proposed MCP algorithm of Section. III-B The simulation considers 10 UEs randomly distributed in the given geographical area. As explained in Section III-B and shown in Fig. 77. $R=455 \mathrm{~m}$ requires one level of circle packing. Using the HPs from the first part of Algorithm 2 as the input, the second part removes the redundant coverage of a UE by different HPs and then discards the HPs with no UEs in its coverage region. As seen in Fig. 8, each UE is covered by only one of the HPs and one of these (HP5, in Fig. 7), having no UEs in its coverage area, is removed, reducing the total number of HPs from 5 to 4 . In addition, the coverage radius, hence the beamwidth, of each $\mathrm{HP}$ is adjusted considering the position of the farthest UE in each coverage region.

\section{B. Cost analysis results}

Fig. 9 shows the variations of the ACS with the UAVs velocity, the DOD of their batteries, the hovering altitude, the data packet sizes $(D)$ to be exchanged between the UAV and the users, and the maximum allowed time between two UAV passages $T_{\mathrm{n}}$. The cost analysis is carried out using the set of HPs from the previous section as input. The hovering time at an HP is determined as the time required to complete the

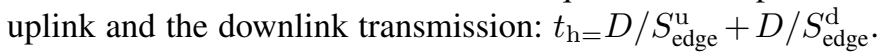
The DOD value that minimizes the annualized cost of the system described in (39) depends on the UAV velocity and $t_{\mathrm{ex}}$. As the curves within the lower black circle in Fig. 9 show, with a small data size (50 Mbits) the velocity should be kept just above $17 \mathrm{~m} / \mathrm{s}$, due to the decrease of $t_{\text {lap }}(v)$ in (41), which in turn decreases the number of UAVs. As velocity increases further, ACS keeps decreasing, albeit with a slowing pace, due to the flying time decreasing, while the hovering time is constant. At even higher velocity the power consumption grows, draining the battery quickly enough to require the deployment of extra UAVs and greater electricity consumption, affecting (40) and (43), and ultimately the ACS. If $T_{\mathrm{n}}$ is large enough (curves with triangles in Fig. 9p, then $t_{\text {lap }}(v) / T_{\mathrm{n}}<1$; hence, the number of active UAVs does not vary with the velocity. Nevertheless, the first component of (41) is velocity-dependant, so the total number of UAVs still varies, although by a small margin. The upper curves in Fig. 9 show what happens with larger data size: the hovering time 


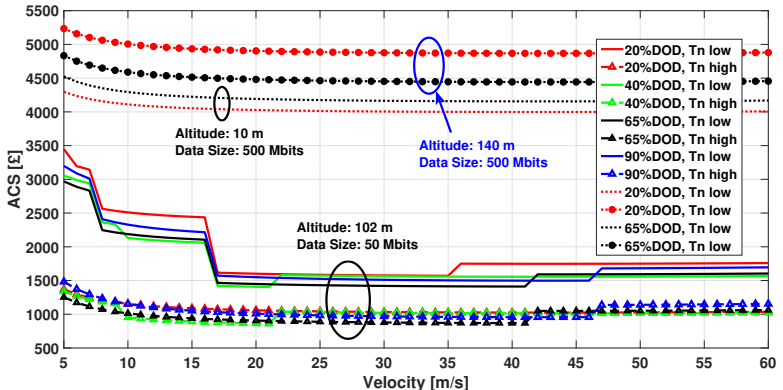

Fig. 9: Cost evaluation with $T_{\mathrm{n}}=100 \mathrm{~s}$ and $T_{\mathrm{n}}=10000 \mathrm{~s}$.

increases, thus the flying time becomes negligible and so does the velocity, resulting in horizontal curves. These curves also show the effect of altitude: since every UAV swap represents a waste of energy and time proportional to $t_{\mathrm{ex}}$, which, in turn, has a positive relationship with the altitude, a higher altitude negatively affects both the number of UAVs and the energy consumption, raising the cost. This effect is more relevant if the DOD is low, as swaps happen more often. The negative effect of higher swap frequency is represented by the gap between the black and the red curve at different altitudes: when the altitude is low (upper black circle) a low DOD results more advantageous, whereas the opposite is true at a higher altitude (blue circle). Therefore, the optimal DOD becomes higher as the mission altitude rises. With reference to (39), the trade-off to determine the optimal DOD is between two opposite forces: maximization of battery lifetime $\left(l_{\text {bat }}\right.$ in (44) $)$ with low DOD; minimization of UAV fleet $\left(n_{\mathrm{u}}\right.$ in (41)) and UAV swaps with high DOD. The latter effects become more relevant as altitude rises, and $t_{\mathrm{ex}}$ with it. Still, since the two forces are comparable, the optimal DOD is unlikely to be extremely high or low, and it must be determined as a function of the given $T_{\mathrm{n}}, D$, and $h_{\text {opt }}$ parameters. With the user distribution shown in Section $\mathrm{V}-\mathrm{A}, T_{\mathrm{n}}=100 \mathrm{~s}$, and a data size of 50 Mbits, the overall cost is minimized when $v=33 \mathrm{~m} / \mathrm{s}$ and the DOD is $50 \%$, resulting in an ACS of $£ 1388$. Fig. 10 shows the variation of

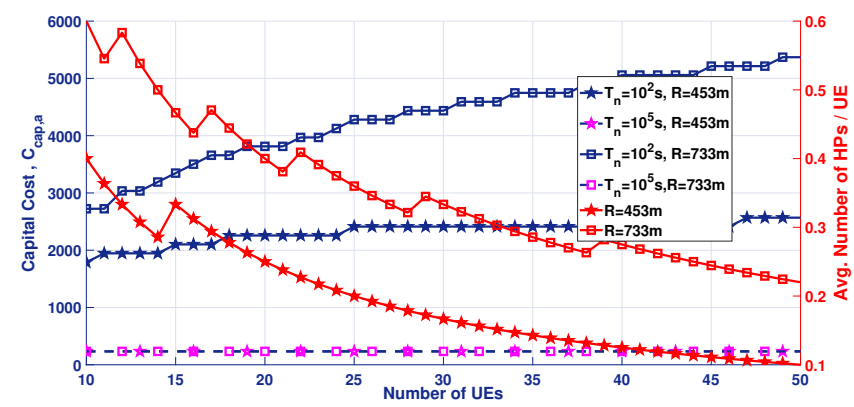

Fig. 10: Capital Cost evaluation with 500 Mbits.

$C_{\text {cap,a }}$ with regards to the number of UEs for different values of $R$. As seen in the figure, the capital cost depends mainly on the delay tolerance of the network: for a high $T_{\mathrm{n}}$ value, $C_{\text {cap,a }}$ is independent of the number of UEs or the $R$-value since a single AAP could fly-hover communicate with the UEs sequentially. Furthermore, the average number of HPs does not increase linearly with the number of UEs because of the efficient $\mathrm{MCP}$ algorithm. Additionally, for a low $T_{\mathrm{n}}$ value,
$C_{\text {cap,a }}$ increases with a decrease in UE density since it needs more HPs to cover widely separated UEs. Hence the system is more cost-efficient when it is deployed to cover a smaller geographical region with a relatively high number of UEs. The 3-D trajectory optimization of an AAP that communicates to a set of mobile users [31], [32] while flying is left for future work.

\section{CONCLUSION}

In this work, we carried out a cost and energy optimization of a UAV-based aerial communication network by taking into account both telecommunications and energy parameters. Observing that the GEE becomes an increasing function of the hovering altitude, the 3-D hovering locations of an AAP that maximize the uplink and downlink GEE are determined using the proposed MCP algorithm. The coordinates generated this way are used as input for the cost analysis, which calculates the number of UAVs needed and how deeply they should exploit their batteries (optimal DOD) and flying parameter (optimal velocity), in order to minimize the annualized total cost of the system. The optimal DOD showed to have an indirect dependency with the altitude, as this extends the UAV swap time $\left(t_{\mathrm{ex}}\right)$. The optimal UAV velocity was calculated for a low data size because its effect becomes less relevant at a higher data size.

\section{REFERENCES}

[1] Erdelj, Milan, and Enrico Natalizio. "UAV-Assisted Disaster Management: Applications and Open Issues", 2016 ICNC Kauai, HI, USA; 1-5.

[2] Fotouhi, Azade, et al. "Survey on UAV Cellular Communications: Practical Aspects, Standardization Advancements, Regulation, and Security Challenges," IEEE Commun. Surv. and Tut. 21.4 (2019): 3417-3442.

[3] N. Babu, K. Ntougias, C. B. Papadias and P. Popovski, "Energy Efficient Altitude Optimization of an Aerial Access Point," 31st IEEE PIMRC, London, United Kingdom, 2020, pp. 1-7.

[4] A. Al-Hourani, S. Kandeepan and S. Lardner, "Optimal LAP Altitude for Maximum Coverage," in IEEE Wireless Commun. Lett., vol. 3, no. 6, pp. 569-572, Dec. 2014.

[5] H. He, S. Zhang, Y. Zeng and R. Zhang, "Joint Altitude and Beamwidth Optimization for UAV-Enabled Multiuser Communications," in IEEE Commun. Lett., vol. 22, no. 2, pp. 344-347, Feb. 2018.

[6] M. Alzenad, A. El-Keyi, F. Lagum and H. Yanikomeroglu, "3-D Placement of an Unmanned Aerial Vehicle Base Station (UAV-BS) for Energy-Efficient Maximal Coverage," in IEEE Wireless Commun. Lett., vol. 6, no. 4, pp. 434-437, Aug. 2017.

[7] H. Liao et al., "Learning-Based Intent-Aware Task Offloading for AirGround Integrated Vehicular Edge Computing," in IEEE Trans. on Intell. Transp. Syst. 2020 Oct 29.

[8] Z. Zhou et al., "When Mobile Crowd Sensing Meets UAV: EnergyEfficient Task Assignment and Route Planning," in IEEE Trans. on Commun., vol. 66, no. 11, pp. 5526-5538, Nov. 2018

[9] M. Mozaffari, W. Saad, M. Bennis and M. Debbah, "Efficient Deployment of Multiple Unmanned Aerial Vehicles for Optimal Wireless Coverage," in IEEE Commun. Lett., vol. 20, no. 8, pp. 1647-1650, Aug. 2016.

[10] Liu, Xiao, et al. "Trajectory design and power control for multi-UAV assisted wireless networks: A machine learning approach," IEEE Trans. on Veh. Technol. 68.8 (2019): 7957-7969

[11] E. Kalantari, H. Yanikomeroglu, and A. Yongacoglu, "On the Number and 3D Placement of Drone Base Stations in Wireless Cellular Networks," in 2016 IEEE 84th VTC-Fall, IEEE, 2016, pp. 1-6.

[12] I. Donevski and J. J. Nielsen, "Dynamic Standalone Drone-Mounted Small Cells," 2020 EuCNC, Dubrovnik, Croatia, 2020, pp. 342-347.

[13] S. Eom, H. Lee, J. Park, and I. Lee, "UAV-Aided Wireless Communication Designs with Propulsion Energy Limitations," IEEE Trans. on Veh. Technol., 2019. 
[14] J. Lyu, Y. Zeng, and R. Zhang, "Cyclical Multiple Access in UAVAided communications: A throughput-Delay Tradeoff," IEEE Wireless Commun. Lett., vol. 5, no. 6, pp. 600-603, Dec 2016.

[15] Wu, Qingqing, and Rui Zhang. "Common Throughput Maximization in UAV-Enabled OFDMA Systems with Delay Consideration," IEEE Trans. on Commun. 66.12 (2018): 6614-6627.

[16] I. Valiulahi and C. Masouros, "Multi-UAV Deployment for Throughput Maximization in the Presence of Co-Channel Interference," in IEEE Internet of Things Journal, vol. 8, no. 5, pp. 3605-3618, Mar., 2021.

[17] Y. Zeng, J. Xu and R. Zhang, "Energy Minimization for Wireless Communication with Rotary-Wing UAV," in IEEE Trans. on Wireless Commun., vol. 18, no. 4, pp. 2329-2345, April 2019.

[18] Y. Zeng and R. Zhang, "Energy-Efficient UAV Communication With Trajectory Optimization," in IEEE Trans. on Wireless Commun., vol. 16, no. 6, pp. 3747-3760, June 2017.

[19] N. Babu, C. B. Papadias and P. Popovski, "Energy-Efficient 3D Deployment of Aerial Access Points in a UAV Communication System," in IEEE Commun. Lett., vol. 24, no. 12, pp. 2883-2887, Dec. 2020.

[20] S. Shakoor, Z. Kaleem, M. I. Baig, O. Chughtai, T. Q. Duong and L. D. Nguyen, "Role of UAVs in Public Safety Communications: Energy Efficiency Perspective," in IEEE Access, vol. 7, pp. 140665-140679, 2019.

[21] L. Chiaraviglio et al., "Minimum Cost Design of Cellular Networks in Rural Areas with UAVs, Optical Rings, Solar Panels, and Batteries," IEEE Trans. Green Commun. Netw., vol. 3, no. 4, pp. 901-918, 2019.

[22] J. Polo, G. Hornero, C. Duijneveld, A. García, and O. Casas, "Design of a low-cost Wireless Sensor Network with UAV mobile node for agricultural applications," Comput. Electron. Agric., vol. 119, pp. 19-32, Nov. 2015.

[23] Li, Ting, et al., "Optimizing the Coverage via the UAVs with Lower Costs for Information-Centric Internet of Things," IEEE Access, vol. 7, pp. 15292-15309, 2019.

[24] 3GPP, "Physical layer procedures,", TR 36.213, Sep. 2015, v 10.12.0.

[25] Tóth, G. Fejes, "Thinnest covering of a circle by eight, nine, or ten congruent circles," Combinatorial and comput. geometry 52.361 (2005).

[26] A. Goldsmith, Wireless communications. Cambridge university press, 2005.

[27] Bilal, B. Ould, et al., "Multi-objective design of PV-wind-batteries hybrid systems by minimizing the annualized cost system and the loss of power supply probability (LPSP)," in 2013 IEEE ICIT, 2013, pp. $861-868$.

[28] C. Bouras, A. Kollia and A. Papazois, "Sensitivity analysis of small cells and DAS techno-economic models in mobile 5G," in 2016 IEEE Wireless Communications and Networking Conference, 2016, pp. 1-6.

[29] N. Omar et al., "Lithium iron phosphate based battery - Assessment of the aging parameters and development of cycle life model," Appl. Energy, vol. 113, pp. 1575-1585, Jan. 2014.

[30] Filippone A, "Flight performance of fixed and rotary wing aircraft", Elsevier; 2006 May 10.

[31] Tang, Jie, et al., "On the Security-Reliability and Secrecy Throughput of Random Mobile User in Internet of Things," in IEEE Internet of Things J., vol. 7, no. 10, pp. 10635-10649, Oct. 2020

[32] Tang, Jie, et al., "Impact of Mobility on Physical Layer Security Over Wireless Fading Channels," in IEEE Trans. on Wireless Commun., vol. 17, no. 12, pp. 7849-7864, Dec. 2018.

Nithin Babu is an early stage researcher at Alba, The American college of Greece for the EU H2020 ITN project PAINLESS and he is enrolled as a $\mathrm{Ph} . \mathrm{D}$ student at Aalborg University, Denmark. He received the B.Tech degree in Electronics and Communication Engineering from CUSAT, India, in 2013 , and the M.Tech degree in Communication Systems Engineering from Indian Institute of Technology, Patna, India in 2016. During his M.Tech, he was a DAAD exchange student at Vodafone chair, TU Dresden and was awarded as the best student in order of merit. His research interests include UAV communication, LoS- MIMO systems, mm-wave communication.

Marco Virgili is an early stage researcher at Lyra Electronics Limited for the EU H2020 ITN project PAINLESS, and he is enrolled as a PhD student at The University of Manchester, UK. He received both a BSc and a MSc degree in Energy Engineering from Sapienza University of Rome, Italy, in 2015 and 2019, respectively. During his MSc, he was an exchange student at TalTech, Estonia, and at University Carlos III of Madrid, Spain. His research interests include power electronics, renewable energy generation, and battery technology. He is currently working on energy-enabling solutions for 5G Base Stations via energy storage system.
Constantinos B. Papadias is the founding Executive Director of the Research, Technology and Innovation Network (RTIN) of The American College of Greece (ACG), in Athens, Greece, and a Professor of Information Technology at ACG's Deree College and Alba graduate school, since Feb. 1, 2020. He is also the Head of RTIN's Smart Wireless Future Technologies (SWIFT) Lab. Prior to these, he was the Dean of Athens Information Technology (AIT), in Athens, Greece, where he was also Head of the Broadband Wireless and Sensor Networks (B-WiSE) Research Group. He currently holds Adjunct Professorships at Aalborg University and at the University of Cyprus. He received the Diploma of Electrical Engineering from the National Technical University of Athens (NTUA) in 1991 and the Doctorate degree in Signal Processing (highest honors) from the Ecole Nationale Supérieure des Télécommunications(ENST), Paris, France, in 1995. He was a researcher at Institut Eurécom (1992-1995), Stanford University (1995-1997) and Bell Labs (as Member of Technical Staff from 1997-2001 and as Technical Manager from 2001-2006).He was also Adjunct Professor at Columbia University (2004-2005) and Carnegie Mellon University (2006-2011). He has published over 210 papers and 4 books and has received over 9500 citations for his work, with an h-index of 44 . He has acted as Technical Coordinator in several EU projects such as: CROWN in the area of cognitive radio; HIATUS in the area of interference alignment; HARP in the area of remote radio heads and ADEL in the area of licensed shared access. He is currently the Research Coordinator of the European Training Network project PAINLESS on the topic of energy autonomous infrastructure-less wireless networks and the Technical Coordinator of the EU CHIST-ERA project FIREMAN on the topic of predictive maintenance via machine type wireless communication systems. His distinctions include the Bell Labs President's Award (2002), the IEEE Signal Processing Society's Young Author Best Paper Award (2003), a Bell Labs Teamwork Award (2004), his recognition as a "Highly Cited Greek Scientist" (2011), two IEEE conference paper awards $(2013,2014)$ and a "Best Booth" Award at EUCNC (2016). He has also been shortlisted twice for the Bell Labs Prize $(2014,2019)$. He was a Distinguished Lecturer of the IEEE Communications Society for 2012-2013. He was appointed Fellow of IEEE in 2013 and Fellow of the European Alliance of Innovation (EAI) in 2019.

Petar Popovski (S'97-A'98-M'04-SM'10-F'16) is a Professor at Aalborg University, where he is heading the section on Connectivity. He received his Dipl. Ing and M. Sc. degrees in communication engineering from the University of Sts. Cyril and Methodius in Skopje and the Ph.D. degree from Aalborg University in 2005. He is a Fellow of IEEE, has over 400 publications in journals, conference proceedings, and edited books and he was featured in the list of Highly Cited Researchers 2018, compiled by Web of Science. He holds over 30 patents and patent applications. He received an ERC Consolidator Grant (2015), the Danish Elite Researcher award (2016), IEEE Fred W. Ellersick prize (2016), IEEE Stephen O. Rice prize (2018) and the Technical Achievement Award from the IEEE Technical Committee on Smart Grid Communications. He is currently a Steering Committee Member of IEEE SmartGridComm and IEEE Transactions on Green Communications and Networking. He previously served as a Steering Committee Member of the IEEE INTERNET OF THINGS JOURNAL. He is currently an Area Editor of the IEEE TRANSACTIONS ON WIRELESS COMMUNICATIONS. Prof. Popovski was the General Chair for IEEE SmartGridComm 2018 and IEEE Communication Theory Workshop 2019. From 2019, he is also a Member-atLarge of the Board of Governors of the IEEE Communications Society. His research interests are in the area of wireless communication, communication theory and Internet of Things. In 2020 he published the book "Wireless Connectivity: An Intuitive and Fundamental Guide".

Andrew J. Forsyth (Senior Member, IEEE) received the B.Sc.(Eng.) degree in electrical engineering from Imperial College, London, U.K., in 1981, and the Ph.D. degree in power electronics from the University of Cambridge, Cambridge, U.K., in 1987. He was a Design Engineer with GEC Electrical Projects, Ltd., from 1981 to 1983, a Lecturer with the University of Bath from 1986 to 1990 , and a Lecturer/Senior Lecturer with Birmingham University from 1991 to 2004. Since 2004, he has been a Professor of power electronics with the University of Manchester, Manchester, U.K. His research interests include high-frequency converters and magnetic components, converter modeling and control, and aerospace and electric vehicle applications. 